\title{
Collaborative Ocean Resource Interoperability - Multi-use of ocean data on the semantic web
}

\author{
Feng (Barry) Tao, Jon Campbell, Maureen Pagnani, Gwyn Griffiths \\ National Oceanography Centre Southampton, U.K. \\ $\{$ bt, joc, gxg, mred\}@noc.soton.ac.uk
}

\begin{abstract}
Earth Observations (EO) collect various characteristics of the objective environment using sensors which often have different measuring, spatial and temporal coverage. Making individual observational data interoperable becomes equally important when viewed in the context of its expensive and time-consuming EO operations. Interoperability will improve reusability of existing observations in both the broader context, and with other observations. As a demonstration of the potential offered by semantic web technology, we have used the National Oceanography Centre Southampton's Ferrybox project (where suites of environmental sensors installed on commercial ships collect near real time data) to set up an ontology based reference model of a Collaborative Ocean, where relevant oceanographic resources, such as sensors and observations, can be semantically annotated by their stakeholders to produce RDF format metadata to facilitate data/resource interoperability in a distributed environment. We have also demonstrated an infrastructure where common semantic management activities are supported, including ontology management, semantic annotation, storage, and reuse (navigating, inference and query). Once the method and infrastructure are adopted by other related oceanographic projects to describe their resources and move their metadata onto the semantic web, it would be possible to see better interoperability within the Collaborative Ocean initiative to facilitate multiuse of ocean data, as well as making more EO data available on the semantic web.
\end{abstract}

Keywords: Semantic Web, data/resource interoperability, oceanographic earth observation, Ferrybox, Multiuse of ocean data

\section{Introduction}

Advances in Web and sensor technologies have made available more and more environmental observations, both remote and in-situ, accessible in real time and as archived datasets. For example, the Ferrybox project [1] at the National Oceanography Centre Southampton (NOCS) has been in operation for six years, making oceanographic observations from various "Ships of Opportunity". One of the Ferryboxes is installed in the engine room of the Pride of Bilbao passenger ferry that sails between the south of England and Northern Spain. The Ferrybox maintains a closed system where near-to-surface sea water is pumped through a set of sensors to carry out observations of various environmental parameters such as temperature, salinity, fluorescence (as an indicator of the quantity of chlorophyll), turbidity, dissolved oxygen and carbon dioxide. Since 2002 a subset of the data collected form the Pride of Bilbao has been automatically telemetered to computers at NOCS every 10 minutes. These data are used to generate near real-time, public web display of ship track and oceanographic measurement information [7]. Datasets are available for downloading and quality-controlled data are eventually archived at British Oceanographic Data Centre (BODC) to allow future data analysis requirement by environmental scientists to understand the change of our natural environment. The Ferrybox project observations are by nature, dense in time coverage but restricted to only a small spatial coverage for a set of limited measurements; Coarse but more global-scale measurements can be obtained through remote sensing observations, from regional aircrafts to earth-orbiting satellites. However, the lack of standardizations between these observations has made it difficult to collate measurements from multiple providers in order to automate intelligent processes, such as measurement cross-validation, data/services mash-up, and personalised knowledge representation based on intelligent processing of distributed measurement data. In other words, the lack of standards and semantics will "isolate important data streams and intensify the existing problem of too much data and not enough knowledge"[2]

On the other hand, as a multi-disciplinary subject, ocean science often involves collaborative work from different stakeholders and roles such as sensor development researchers, oceanographic instrument deployment engineers, data management, and environmental scientists, each of whom provides, as well as 
consumes data/services that are shared within the domain. Within the context of Oceans 2025, which is a strategic marine science programme funded by the UK Natural Environment Research Council (NERC), bringing together marine researchers to increase people's knowledge of the marine environment so that they are better able to protect it for future generations [13]. For example, in the Ferrybox project, engineers need to collaborate with the sensor providers in order to select and integrate sensors into the Ferrybox Instruments, which are then deployed on platforms (e.g. Ships of Opportunity in this case). Observations are sampled and brought back regularly by telecommunication though satellite and on-site visits to collect the physical memory storage. Real time monitoring is used to monitor the Ferrybox status. Calibrations for sensors are inevitably needed as the measurements drift due to bio-fouling and other reasons, hence their calibration history should be recorded to allow easy management for future reuse. Datasets of the observations are then archived by data managers with various metadata and descriptions attached so that domain scientists can investigate a particular environmental phenomenon, e.g. a bloom of plankton and its relationship with temperature and salinity [8]. All these activities through out the workflow have access to different yet related resources which can be annotated against a domain reference model to reduce ambiguity (every resource has a unique formal identifier) and improve connectivity (resources are linked to other resources through defined relations). This eventually will support sharing earth observation data and knowledge.

\subsection{Related work and motivation}

The Semantic Sensor Web (SSW) is a framework for providing well defined meaning using the semantic web technology in W3C that realises the idea of "having data on the Web defined and linked in a way that it can be used by machines not just for display purposes, but for automation, integration, and reuse of data across various applications" [4]. The underpinning modelling language for the semantic web is the RDF (Resource Description Framework), which is a directed-graph model using XML syntax that can also be expressed in many other forms, e.g. Notation3 (N3). The RDF data model describes the real world by making statements in the form of triples (subject-predicate-object) where every item is either a literal or a URI (Uniform Resource Identifier) prefixed with a name space. RDF is an open system in that the namespaces can be unlimited, each one corresponding to an ontology that defines a formal conceptual representation within a domain - concepts and relationships in particular. The simple RDF data model allows computers to store, exchange, and to some extent reason (think!) using information from across the Web, providing opportunities for computer-assisted information handling with more assurance and certainty.

Semantic Web based knowledge management has been previously introduced and successfully applied in engineering design search optimization [14] and e-learning [15]. Both domains are single core interdisciplinary, i.e. between computer science and one more area. The same application in environmental domain is multi-core inter-disciplinary area where the domain is more diversified to include for example oceanography, electronics, chemistry, biology, etc. Therefore setting up the infrastructure and gluing/reusing existing domain ontology are more important.

With these objectives in mind, we have created a Collaborative Ocean Group (COG) in the context of Marine Metadata Interoperability (MMI) [5] to demonstrate this proof of concept for multi-use of ocean data. In order to coordinate oceanographic activities and facilitate resource sharing and data interoperability, we describe in this article a semantic web infrastructure that helps different roles to semantically annotate their resources so that they can be managed as linked-data which are W3C compatible to facilitate semantic web processing.

Ferrybox has been used as our first example to help us understand the domain, building up a domain reference model in an ontology, and to test drive the semantic web infrastructure for Collaborative Ocean Resource Interoperability. In this work, we have created a conceptualisation of the domain through using existing vocabularies as well as creating our domain specific ontologies.

\section{Ontology based metadata modeling}

Metadata has been widely used in environmental data management systems to improve both the accessibility and the quality of the observational information. However, interoperability between these systems is often restricted due to the lack of semantics and closeness of their metadata reference models. In our work, we aim 
to improve the situation by adopting the Semantic Web approach. For example, the concepts of namespace and URI in the Semantic Web make it possible to describe resources in an open and globally accessible manner. Further more, recent developments in the Semantic Web community have made available more tools and APIs that can handle data on the semantic web for parsing, querying and inference.

\subsection{An ontology based reference model for Collaborative Ocean}

Ontology is a formal representation of a set of commonly agreed concepts and defined relationships between them. It provides shared but controlled vocabularies, makes domain assumptions explicit and can be used to describe resources in a domain in a way that all domain users can collaborate with each other. In short, it is an accepted consensus of domain conceptualisation based on which semantics can be added to enrich domain resources.

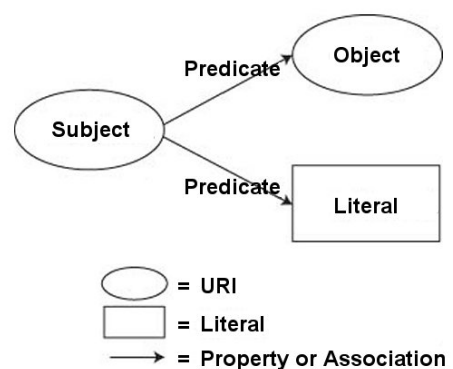

Figure 1 RDF direct graph model

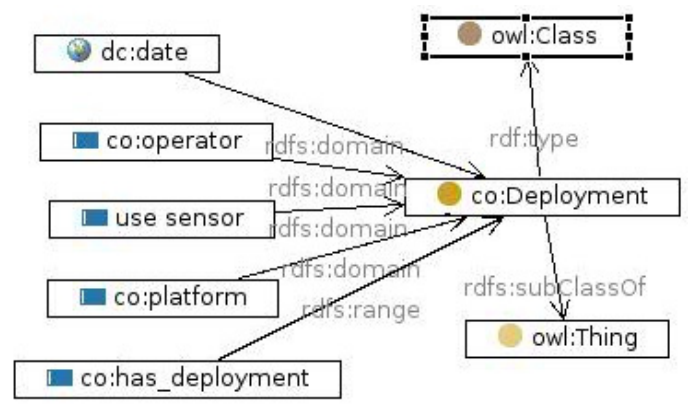

Figure 2 An example in the Ferrybox project

RDF and OWL has been the main model for expressing ontology. The RDF data model is based on the idea of making statements about web resources in the form of subject-predicate-object triples, where each entity in the triple is either a literal or a RDF resource with a URI (Uniform Resource Identifier). A set of triples using RDF resources can be viewed as a directed RDF graph. OWL (Web ontology language) extends the RDF Schema with additional vocabulary, e.g. on property restrictions (allValuesFrom) and restricted cardinality (minCardinality), etc.

\begin{tabular}{|c|c|c|c|}
\hline \multirow[t]{5}{*}{$\begin{array}{l}\text { Upper Level Ontology } \\
\text { (generic) }\end{array}$} & dc (Dublin Core) & http://purl.org/dc/elements/1.1/ & $\begin{array}{l}\text { metadata for web document } \\
\text { and media }\end{array}$ \\
\hline & $\begin{array}{l}\text { rdf (Resource Description } \\
\text { Framework) }\end{array}$ & $\begin{array}{l}\text { http://www.w3.org/1999/02/22 } \\
\text {-rdf-syntax-ns\# }\end{array}$ & $\begin{array}{l}\text { Generic resource description } \\
\text { framework }\end{array}$ \\
\hline & owl & $\begin{array}{l}\text { http://www.w3.org/2002/07/o } \\
\text { wl\# }\end{array}$ & vocabulary extension of RDF \\
\hline & Xsd (W3C XML schema) & $\begin{array}{l}\text { http://www.w3.org/2001/XML } \\
\text { Schema\# }\end{array}$ & $\begin{array}{l}\text { XML Schema definition of } \\
\text { value types, such as integer, } \\
\text { float, string or dates, etc. }\end{array}$ \\
\hline & $\begin{array}{l}\text { Skos (Simple Knowledge } \\
\text { Organization System) }\end{array}$ & $\begin{array}{l}\text { http://www.w3.org/2004/02/sk } \\
\text { os/core\# }\end{array}$ & $\begin{array}{l}\text { An application of RDF that } \\
\text { helps denoting classification, } \\
\text { taxonomy typed relationships }\end{array}$ \\
\hline \multirow[t]{2}{*}{ Middle level ontology } & Foaf (Friend of A Friend) & http://xmlns.com/foaf/0.1/ & $\begin{array}{l}\text { Another RDF application as a } \\
\text { machine readable ontology for } \\
\text { describing Social Network } \\
\text { related entities such as person } \\
\text { and their relationships }\end{array}$ \\
\hline & $\begin{array}{l}\text { Geo (W3C Basic Geo } \\
\text { Vocabulary) }\end{array}$ & $\begin{array}{l}\text { http://www.w3.org/2003/01/ge } \\
\text { o/wgs84_pos\# }\end{array}$ & $\begin{array}{l}\text { Definition of simple geospatial } \\
\text { related properties such as lat } \\
\text { and long }\end{array}$ \\
\hline Domain ontology & $\begin{array}{l}\text { Co (Collaborative Ocean } \\
\text { Ontology) }\end{array}$ & $\begin{array}{l}\text { http://www.soton.ac.uk/ bt/on } \\
\text { tology/ocean/collaberativeocea } \\
\text { n.owl\# }\end{array}$ & $\begin{array}{l}\text { Domain reference model } \\
\text { ontology that glue together } \\
\text { other ontologies }\end{array}$ \\
\hline
\end{tabular}




\begin{tabular}{|l|l|l|l|}
\hline & $\begin{array}{l}\text { Do (Marine Metadata } \\
\text { Interpretability (MMI) Device } \\
\text { Ontology) }\end{array}$ & $\begin{array}{l}\text { http://marinemetadata.org/files } \\
\text { /mmi/OntologyExampleOMV. } \\
\text { owl }\end{array}$ & $\begin{array}{l}\text { Ontology that focus on } \\
\text { environmental observation } \\
\text { sensors }\end{array}$ \\
\hline
\end{tabular}

Table 1 Ontology Types

The ontologies used can be categorized into three types according to their levels of generic description to the domain, as shown in Table 1.

\section{Upper level and meta ontology}

There are many generic conceptualisations that are domain independent and therefore can be used to provide cross-domain resource description.

DC - Dublin Core is a cross-domain XML/RDF based metadata standard designed to describe general media on the web to make them easier to find. It is divided into two levels - Simple Dublin Core (Title, Creator, Date, Subject, etc) and Qualified Dublin Core (Provenance and Rightsholders, etc). We use the DC to describe generic attributes of Ferrybox resources such as datasets collected and put online.

W3C RDF - defined internal vocabulary for RDF, such as rdf:resource and rdf:datatype, etc.

W3C RDFS - this is the root vocabulary and syntax specification originally defined in the RDF model. We use rdfs:seeAlso to maintain linkages between our parameters and BODC controlled vocabularies.

SKOS - noted as Simple Knowledge Organization Schema, it is designed to express generic relationship before concepts, such as skos:broader, skos:related, etc. BODC uses SKOS to express relationship among their control vocabularies.

\section{Middle Level ontology}

These ontologies are less generic than the upper level ontology but more generic than the overall domain ontology.

A common feature of EO data is its geospatial properties. Almost all EO measurements are related to location. We adopted the widely use $\mathrm{W} 3 \mathrm{C}$ GEO vocabulary for this modelling requirement.

Another feature is the inclusion of social network related properties which are often required to describe the different roles in oceanography in such a way as to allow them to communicate and collaborate with each other in order to carry out EO tasks. We adopted the FOAF ontology that is originally proposed in W3C to describe social network related properties.

Here is an example that uses both FOAF and GEO in a RDF file.

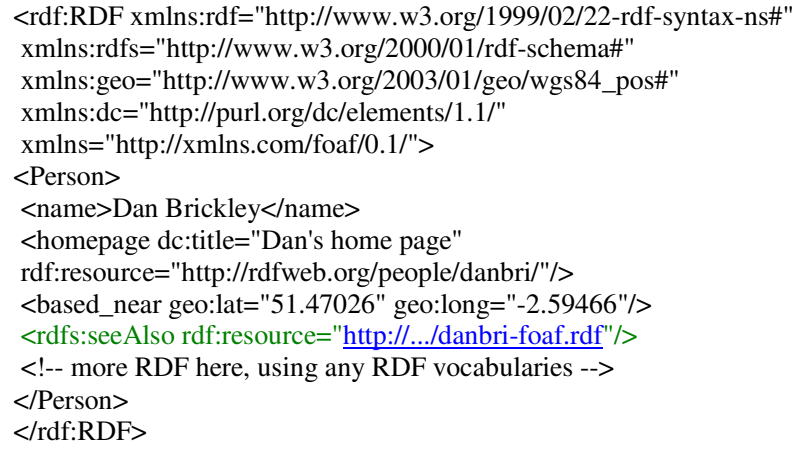

\section{Domain ontology}

Domain ontology is specific to the domain application and is normally non-transferring for other different domains. It is normally acquired by investigating the domain. 


\section{CO - Collaborative Ocean ontology}

This is the main specific reference model ontology designed to provide domain conceptualisation needed for describing domain related resource annotations. The ontology has the namespace "co" and focuses on concept taxonomy (Figure 4) and how they are connected to other concepts through defined properties (Figure 3).

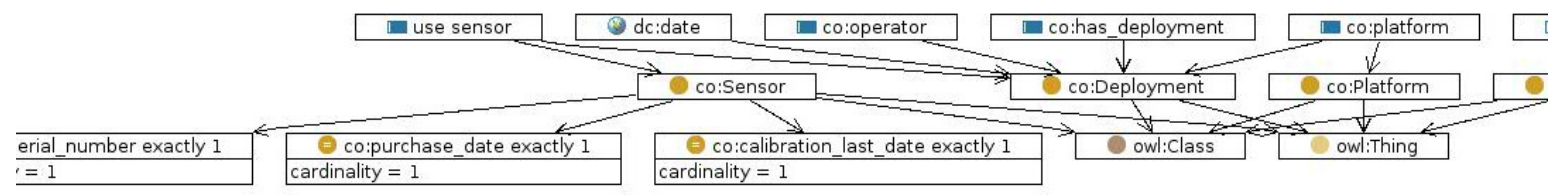

Figure 3 a section of the CO ontology

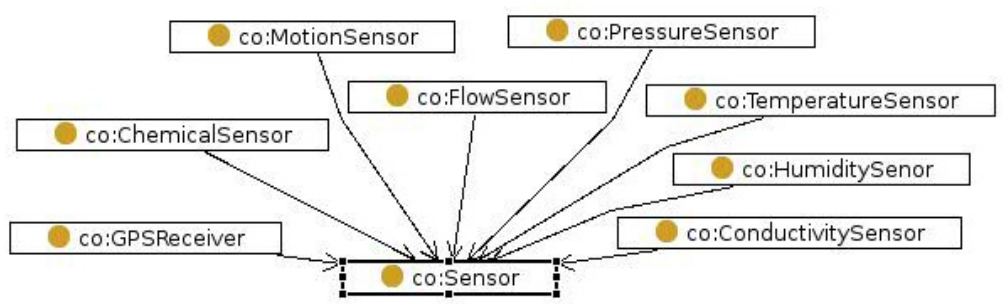

Figure 4 A sensor taxonomy

Some of the important concepts are Sensor, Platform, Deployment, Datasets, etc. RDFS and OWL has been used to explicitly describe concept taxonomy, relationship and constraints in term of other define properties.

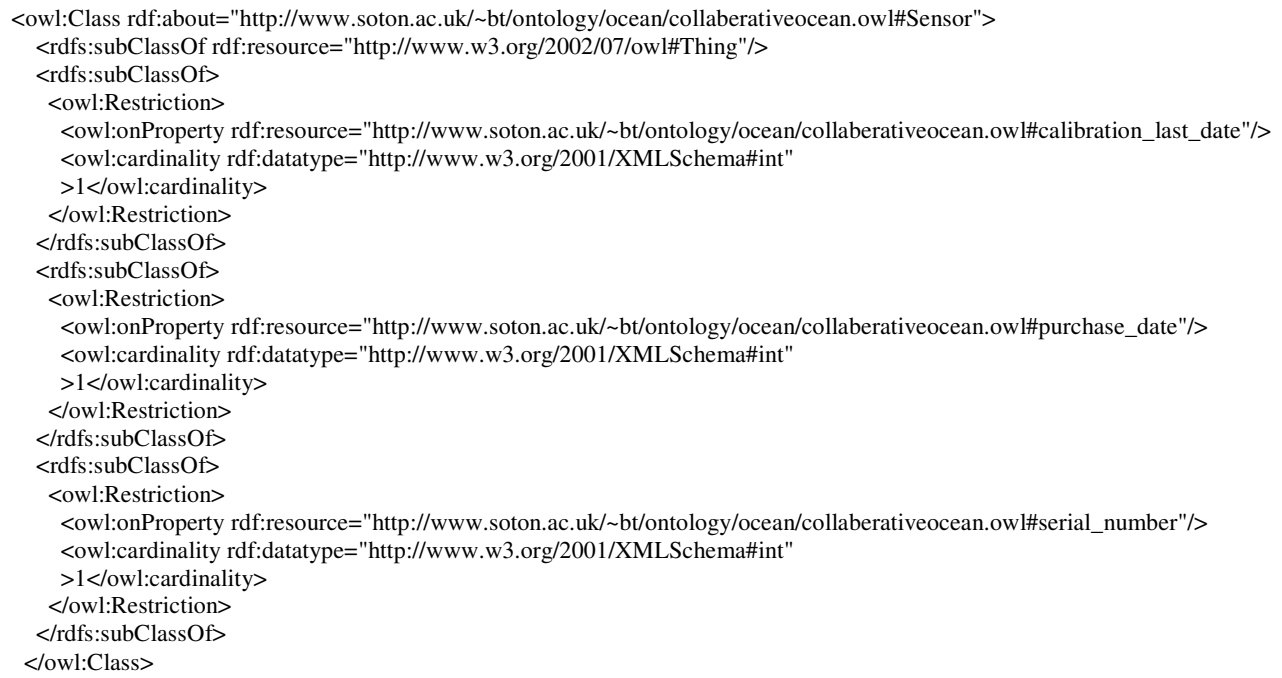

\subsection{Interoperability with existing reference model}

Interoperability with other reference models such as MMI device ontology and BODC vocabulary is maintained by crossed linkage and references. One example is shown below where we have reused BODC measurement parameter vocabulary by adding SKOS annotations. This would provide the semantic linkage between two reference models when navigating on the semantic web from one to the other. 
$<$ co:ChemicalParameter

rdf:about="http://www.soton.ac.uk/ bt/on tology/ocean/collaberativeocean_ins.rdf\#s alinity">

$<$ rdfs:seeAlso

rdf:datatype="http://www.w3.org/2001/X

MLSchema\#string"

>http://vocab.ndg.nerc.ac.uk/term/P021/2

5/PSAL</rdfs:seeAlso >

$<$ co:unit

rdf:datatype="http://www.w3.org/2001/X

MLSchema\#string"

$>$ lu2030</co:unit>

$<$ co:unit xml:lang="en" $>$ ppt $</$ co:unit $>$

$</$ co:ChemicalParameter $>$

* RDF snippet that links to BODC vocabulary

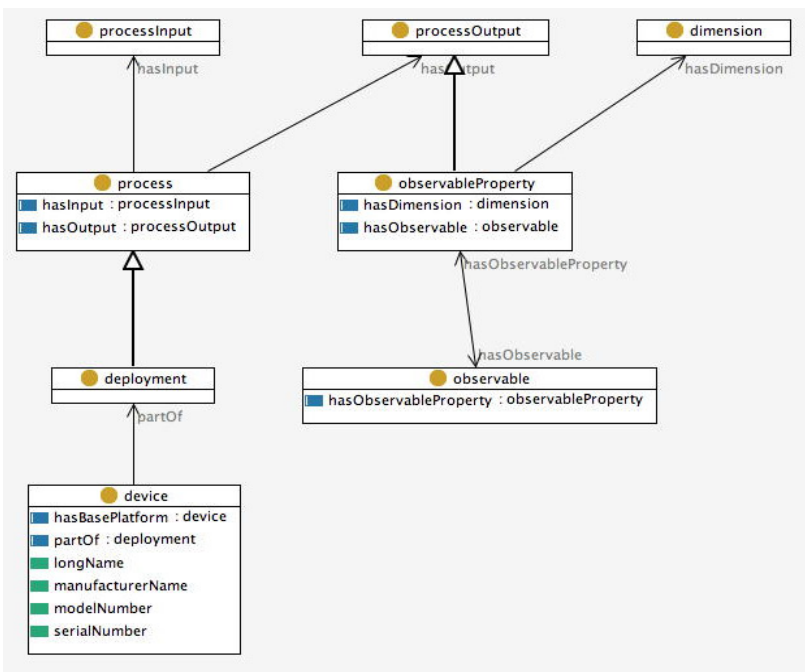

Figure 5 MMI Device ontology

In parallel with the development of the Collaborative Ocean ontology, we have also participated in the MMI Device Ontology Working Group, with an aim to contribute our experience and requirement for sensor annotation metadata and to eventually reuse their device ontology in the CO ontology. This will provide end users with more choices to carry out their sensor device semantic annotation and better enable interoperability in a broader EO context. Figure 5 is a snapshot of the MMI device ontology concept diagram in development.

\section{A semantic web infrastructure for the Collaborative Ocean}

In order to efficiently describe, store and reuse the oceanographic EO operational resource and measurement data on the semantic web, we have set up a layered infrastructure as shown in Figure 6. An advantage of this structure is to allow distributed management of the semantic web information at different granularities, and to expose a different level of technical details to different roles through customized interfaces and toolkits.

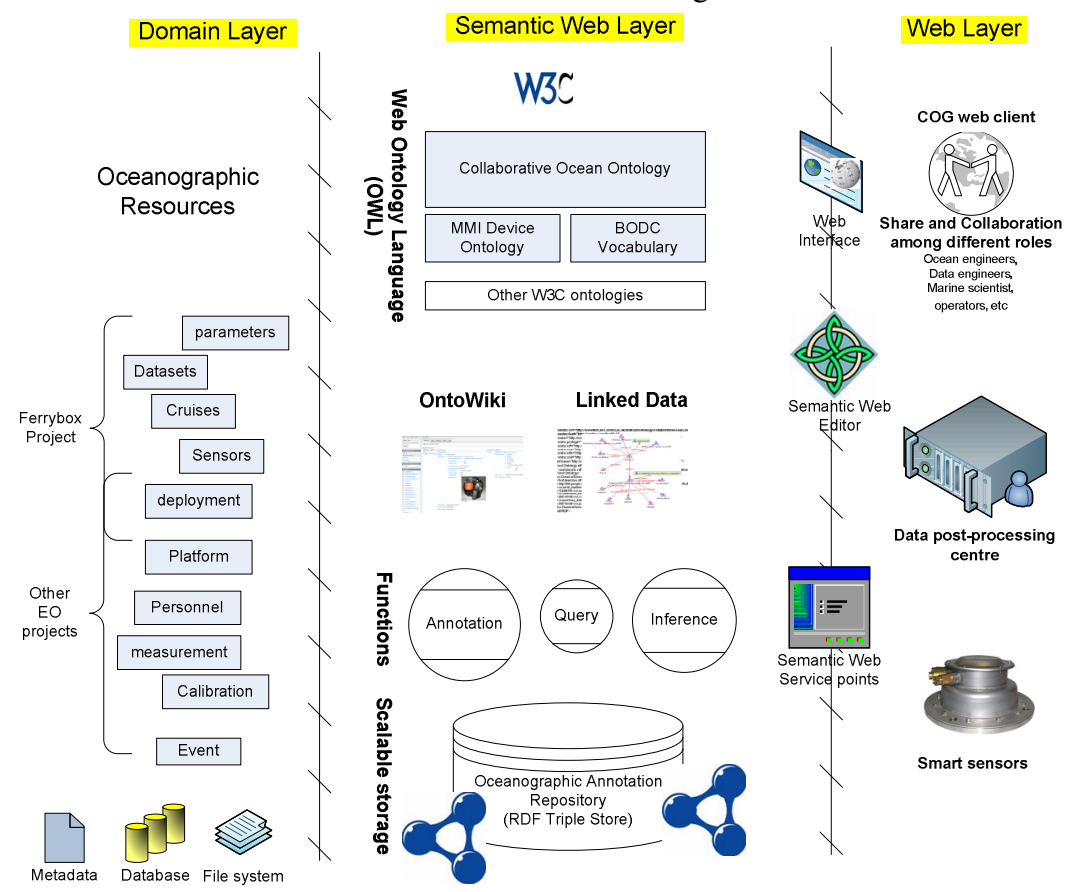

Figure 6 Layered diagram of the semantic web infrastructure 


\section{Domain layer}

Domain resources such as measurement datasets, calibration reports, and sensor profiles as well as existing metadata are stored in this layer, in the form of flat files or database records. The Collaborative Ocean Ontology and relevant controlled vocabulary are modelled and adopted based on study of the resources in this layer as well as best practice directly from experts operating the Ferrybox project. We use not only existing metadata such as Dublin Core and FOAF but also related domain vocabulary such as MMI device ontology and BODC vocabulary. The CO ontology serves as the domain reference model that provides important concepts and assumptions of the domain resource conceptualisation to allow proper semantic annotation of the Ferrybox resources and their publishing on the semantic web. Collaborative Ocean ontology has been built using Collaborative Protégé [9], which is an extension of the existing Protégé system that supports collaborative ontology editing. The Ontology is further maintained in the Topbraid Editing environment [10] to exploit its advantage in managing multiple ontology namespaces and its RDF triple representation of the ontology as well as the semantic annotations simulation. The advantage of Topbraid composer over protégé is its capability in managing multiple external namespaces and accessibility to the openRDF triple store.

During the Knowledge Acquisition (KA) process, we have adopted an agile knowledge modelling method that encourages domain experts to participate in the KA as an iterative process. A Wiki page has been set up to assist recording sensor profile text as well as key description of resources available in the Ferrybox project. A Protégé server has been setup to drive a collaborative protégé client to collect concepts and resource taxonomy from various domain experts in the group for a very initial version of the CO ontology. As shown in Figure 7, the RDF data is then fed into an OntoWiki [11] web based knowledge acquisition system as an information map where intuitive authoring of semantic RDF content as well as simulation of the semantic annotation process is made possible to enhance understanding of the domain. The OntoWiki adopted an agile and adaptive knowledge engineering strategy that is similar to a wiki-style paradigm of "making it easy to correct mistakes, rather than making it hard to make them" [12]. The Semantic Wiki is another similar tool that integrates the Semantic Web into a Wiki system. Interested users can find a recent review at [18]
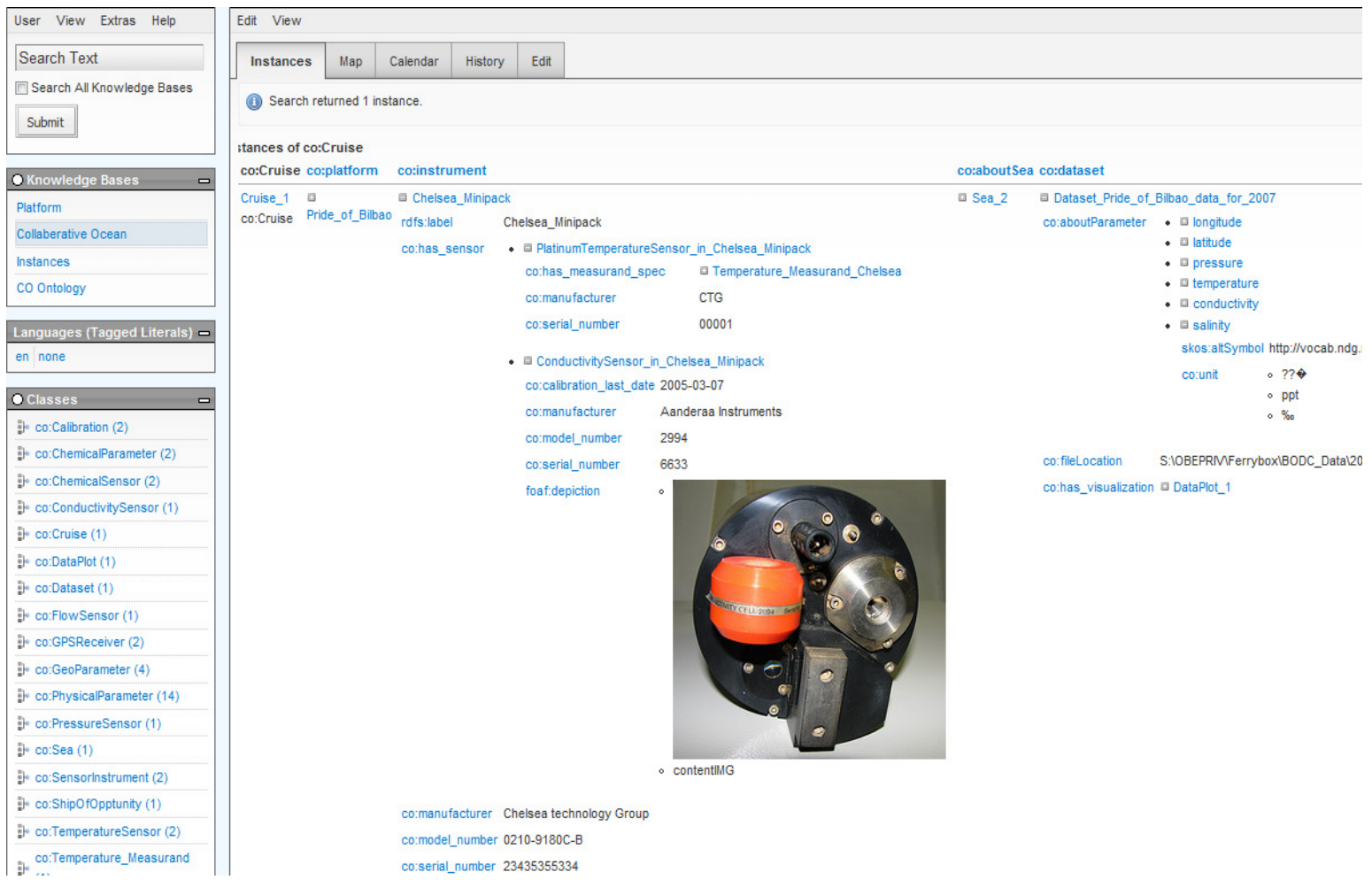

Figure 7 OntoWiki interfaces 


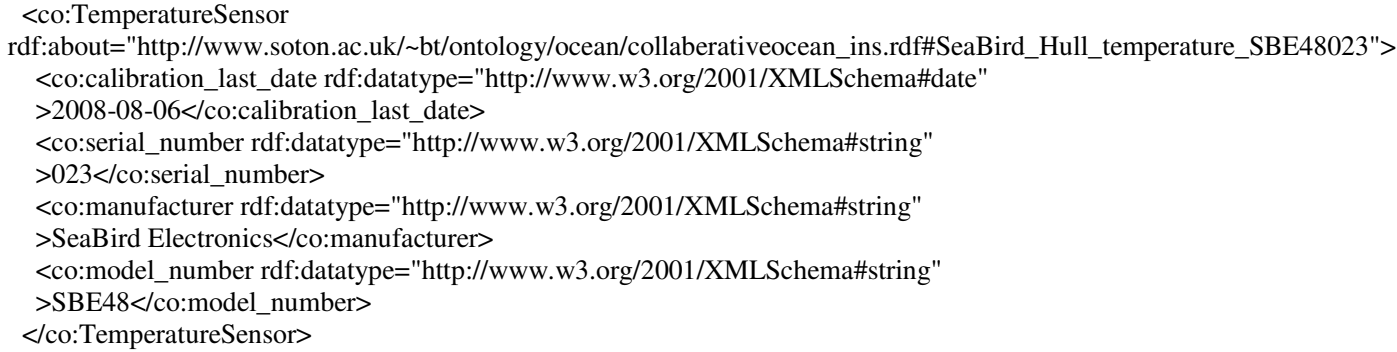

\section{Web layer}

This is where applications are deployed to interface end users with the functionalities offered by the semantic web layer in the context of the domain. We have implemented a COG web client that allows manual and semi-automated semantic management of oceanographic resource share and collaboration using semantic web functionalities offered in the semantic web layer of the system. More intelligent, semantic web driven operations, such as data post-processing and smart sensor activities can be also supported in the future.

\section{Technical implementation and how the system works}

The system components/services have been deployed onto multiple nodes of different operating systems (Windows XP and Linux) to demonstrate a distributed Service Oriented Architecture (SOA). We have chosen to use Java and PHP as the main development languages due to the fact that most semantic web APIs (such as Jena and Sesame) are written in Java and its portability in different systems. PHP is another popular Web language especially suitable for large scale distributed systems across the Web. We have used Apache and Tomcat to run the web server and provide a Java servlet container where various services are deployed, such as an ontology driven Java Facelet web interface service that invokes semantic web functionalities such as query and inferences to interact with the semantic information in the RDF triple store. The triple store is a Sesame openRDF repository package deployed in another node across the network, where OntoWiki is also deployed to offer an alternative access to the semantic information.

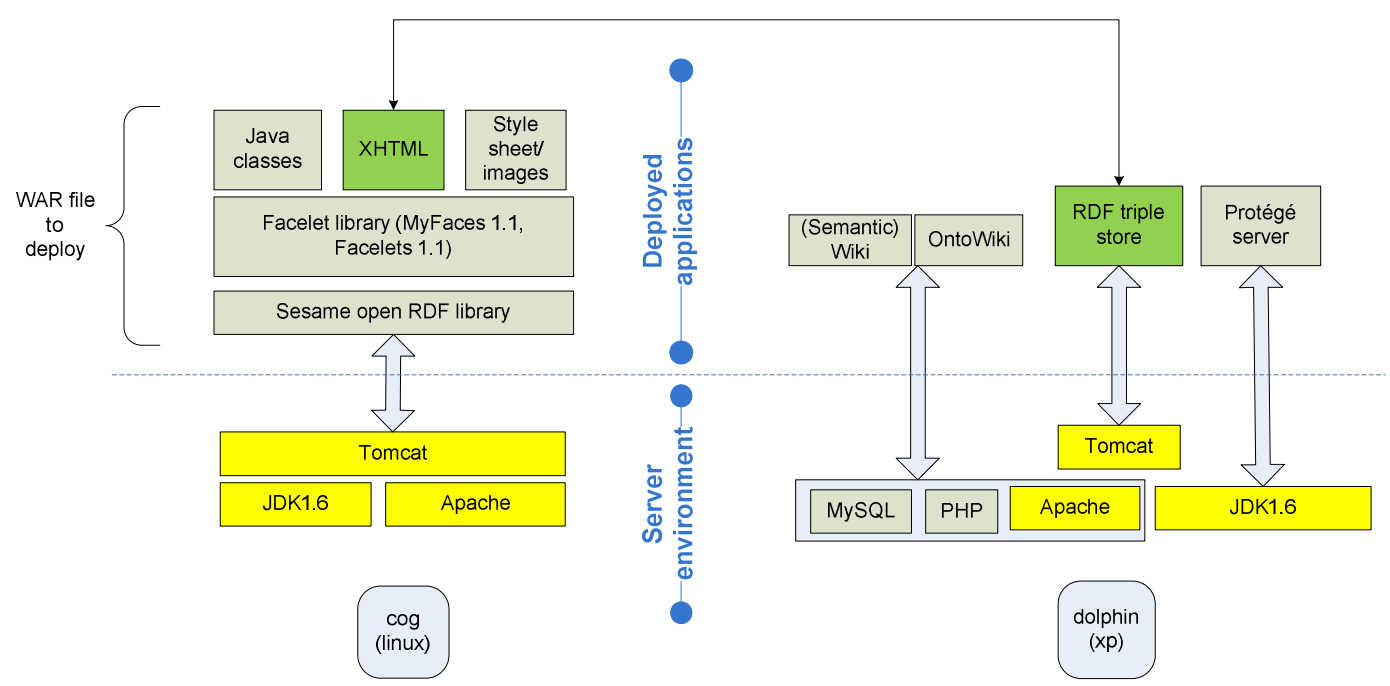

Figure 10 Technical architecture of the system 


\section{Connecting to the system}

A Sesame server address is required to specify the location where all the semantic annotations are stored as RDF triples. Multiple repositories can co-exist in the same server to store the RDF triples.

The user can also indicate whether inference is enabled. The inference is at the RDFS level to include inferred new triples according to RDF schematic reasoning rules. More interesting domain rules can be designed to produce inferred triples based on domain business logics.
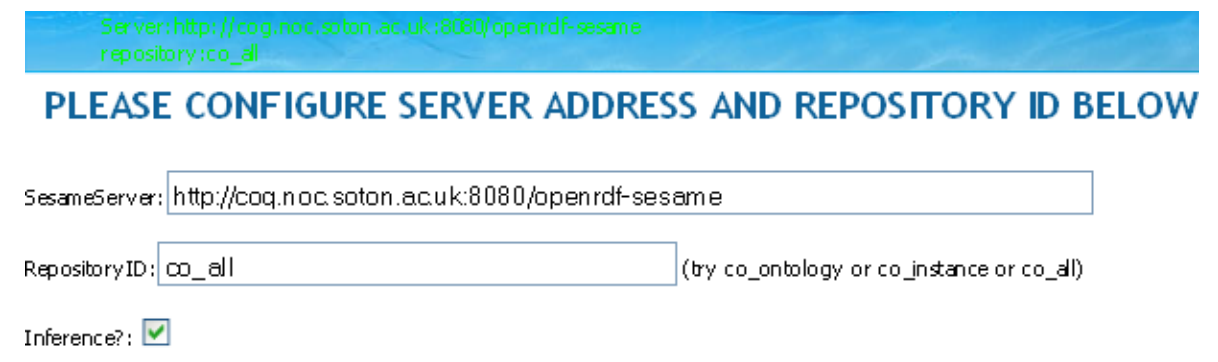

Submit

Figure 11 connecting the RDF triple store

\section{Dynamic ontology driven semantic annotation of domain resources}

Most initial semantic annotations are entered through other channels by our domain experts using Protégé and Topbraid Semantic web toolkits. Once they are stored as RDF triples in the repository, other interested users will be able to reuse part of these triples to generate further semantic annotations in a web interface. Meanwhile, reasoners can be used to generate further RDF statements that represent inference closure results of existing triples.

The web based semantic annotation page is dynamically generated by querying the $\mathrm{CO}$ ontology using SPARQL, therefore it is adaptive during the maturing the CO ontology. SPARQL is the querying language standard for collecting data from the semantic web. It is similar to the SQL for database, but with added semantic web features based on the RDF model and multi-source RDF query across the Web. Below is a SPARQL query for defined properties of the domain class co:ChemicalSensor, used to dynamically generate the semantic annotation forms as shown in Figure 12.

SELECT DISTINCT ?property WHERE $\{$ \{?property rdfs:domain ?class_X\}

$\{$ co:ChemicalSensor rdfs:subClassOf ?class_X \}

UNION \{?property rdfs:domain co:ChemicalSensor\} \}

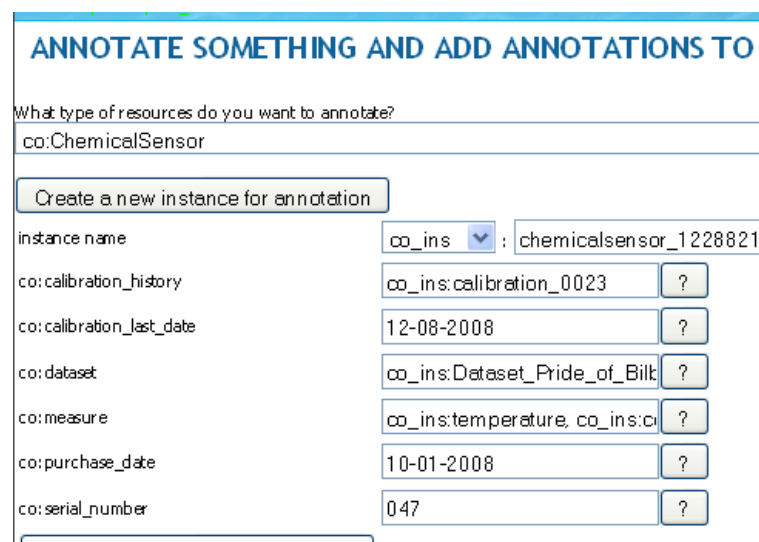

Save annotations to the triple store

Figure 12 ontology driven semantic annotation web form generation

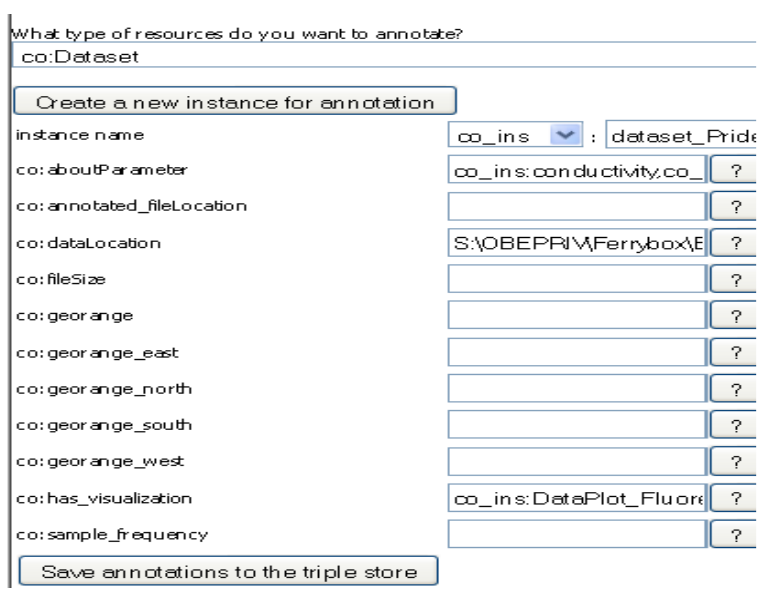

Figure 13 Semantic annotating a Dataset to include co_ins:DataPlot_xxx 
With the ontology driven annotation form, users can annotate datasets by providing values for a set of properties as defined in the ontology. These annotations will be converted as RDF triples and stored in the semantic web triple store so that other users can access through further query or browsing.

For example, as shown in Figure 13, related data visualization of the dataset can be added as part of the semantic annotation to link interesting data plots generated from the dataset. This is done by using "co:has_visulization" which is defined in the $\mathrm{CO}$ ontology as a valid annotation property for Dataset. As noted as "co_ins:DataPlot_xxx" which shows a visualization of annual sea water measurement, such as fluorescence and temperature.

\section{Semantic search, browsing and navigation}

Semantic search, browsing and navigation of the annotations can also be achieved through submitting SPARQL semantic queries to the triple store and rendering results as linked data [19] on the web. Users will be able to view a list of semantically enriched instances of a particular class. They can click on any interesting element of the semantic annotations, for which the system will generate a new query accordingly, and get back another set of matched triples for rendering on the web. As all the resources can be annotated by their stakeholders at any time, these changes will be reflected in other semantic web management activities that reuse these triples.

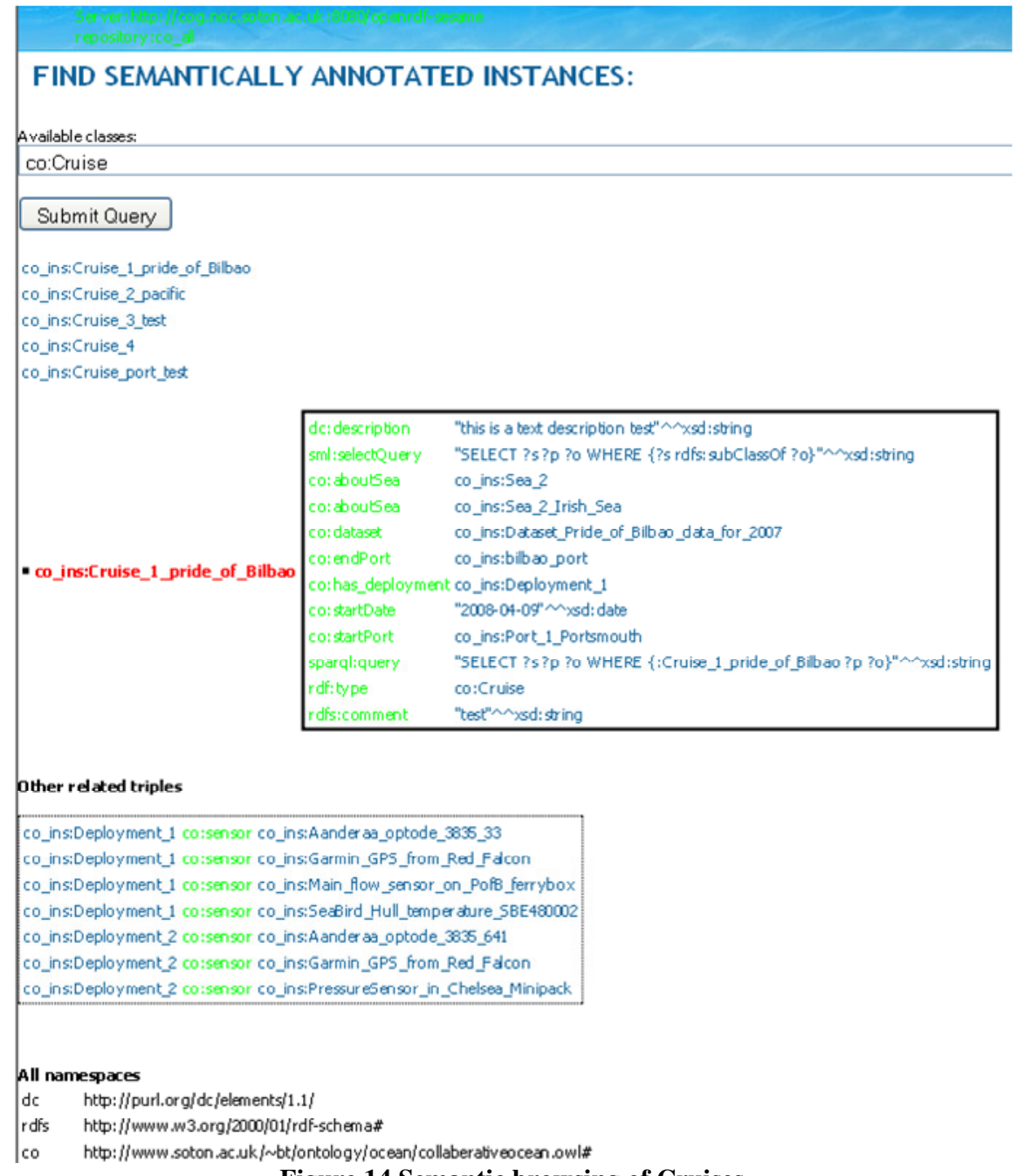

Figure 14 Semantic browsing of Cruises 
Two examples of the semantic search and navigation are illustrated in Figure 14 and Figure 15. According to the semantic annotations, the Ferrybox deployments are associated with the "co:Cruise" concept where additional properties such as "co:aboutSea", "co:startPort" and "co:dataset" can be assigned with values during the semantic annotation process. The semantic query retrieves a list of annotated cruise instances on which users can further browse their semantics and navigate to other linked semantic annotations.

Similarly, queries to dataset annotations can help users to discover their linkages to other annotated resources in the reference model, such as measurement parameters, original dataset producers and visualization plots generated, etc.

A notable feature is the resolvable namespace prefixes that point to corresponding published ontologies that define the underlying semantics.

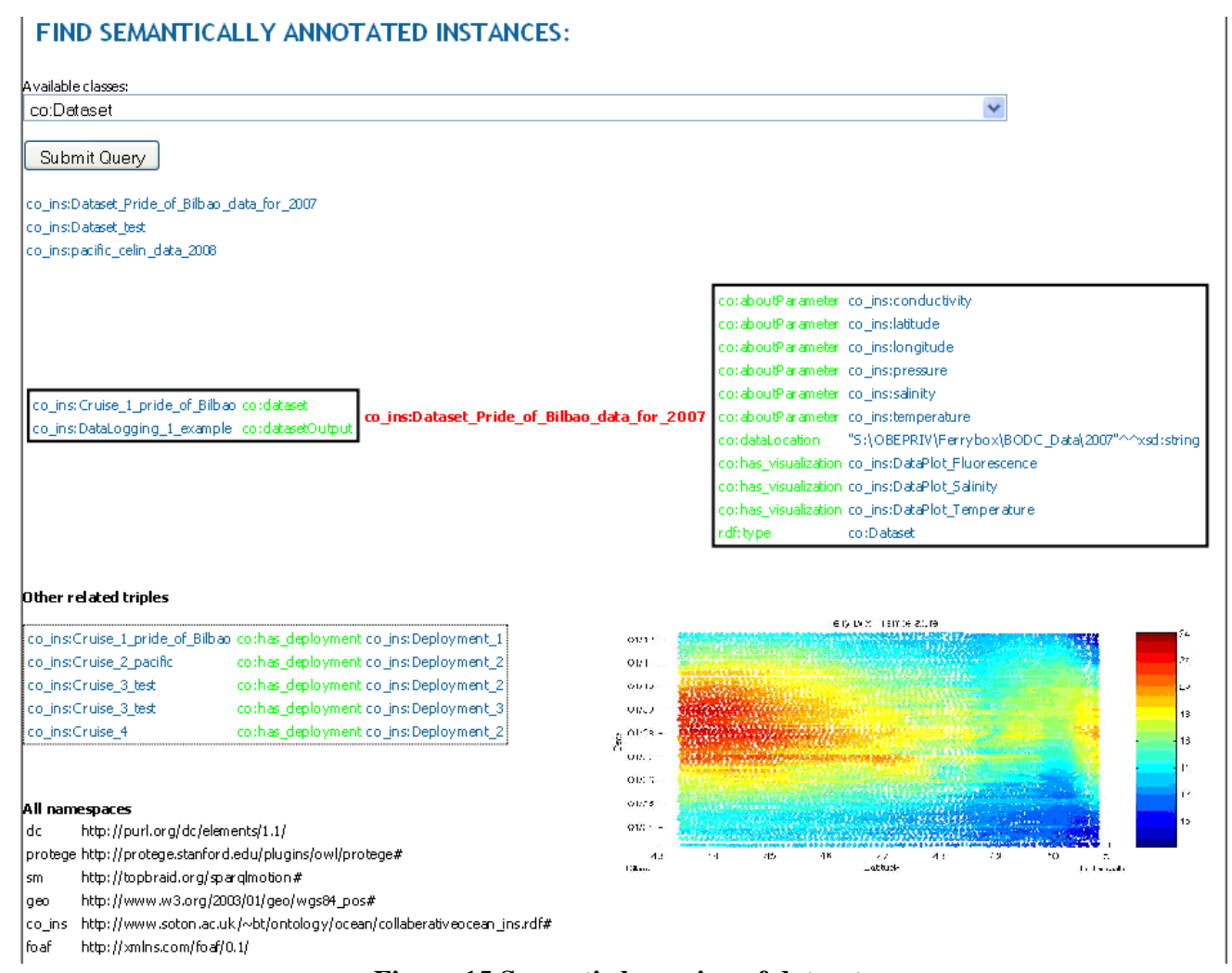

Figure 15 Semantic browsing of datasets

\section{Mashing up with OGC service}

Since the establishment of the Open Geospatial Consortium (OGC) in 1994, a lot of standards and services have been developed to coordinate the geospatial industry all over the world. One of the most relevant initiatives in the OGC is Sensor Web Enablement (SWE), which aims to enable real-time integration of heterogeneous sensor observations into the Web infrastructure. The main specifications include Sensor Model Language (SensorML), Sensor Observation Service (SOS), Sensor Planning Service (SPS), etc [6]. Various XML schemas are defined in SWE to accommodate XML files used in service in/output, e.g. in SOS, SensorML and Transducer Markup Language (TML) are used in describeSensor method response. The getObservation method has an input XML schema that uses Geographical Markup Language (GML) to specify time-spatial coverage of the observations. Observation offerings in SOS also provide non-overlapping observations constrained by a number of parameters such as geographical region and phenomena being sensed. This allows easy integration of spatially referenced data to layers in Web Map Service (WMS), another important OGC specification. 
In the Ferrybox context, we have done some experiments to demonstrate that some resource annotations, such as those of the sea ports, which have been semantically annotated using geospatial metadata, can be queried and their results mashed up with third-party WMS services such as Google map to visualize their geospatial presence. This demonstrates interoperability potential between RDF triples and OGC standard. We believe that as more measurement data are annotated with geospatial references, the mashing up will provide more interesting integration between the $\mathrm{W} 3 \mathrm{C}$ semantic web and the OGC services.

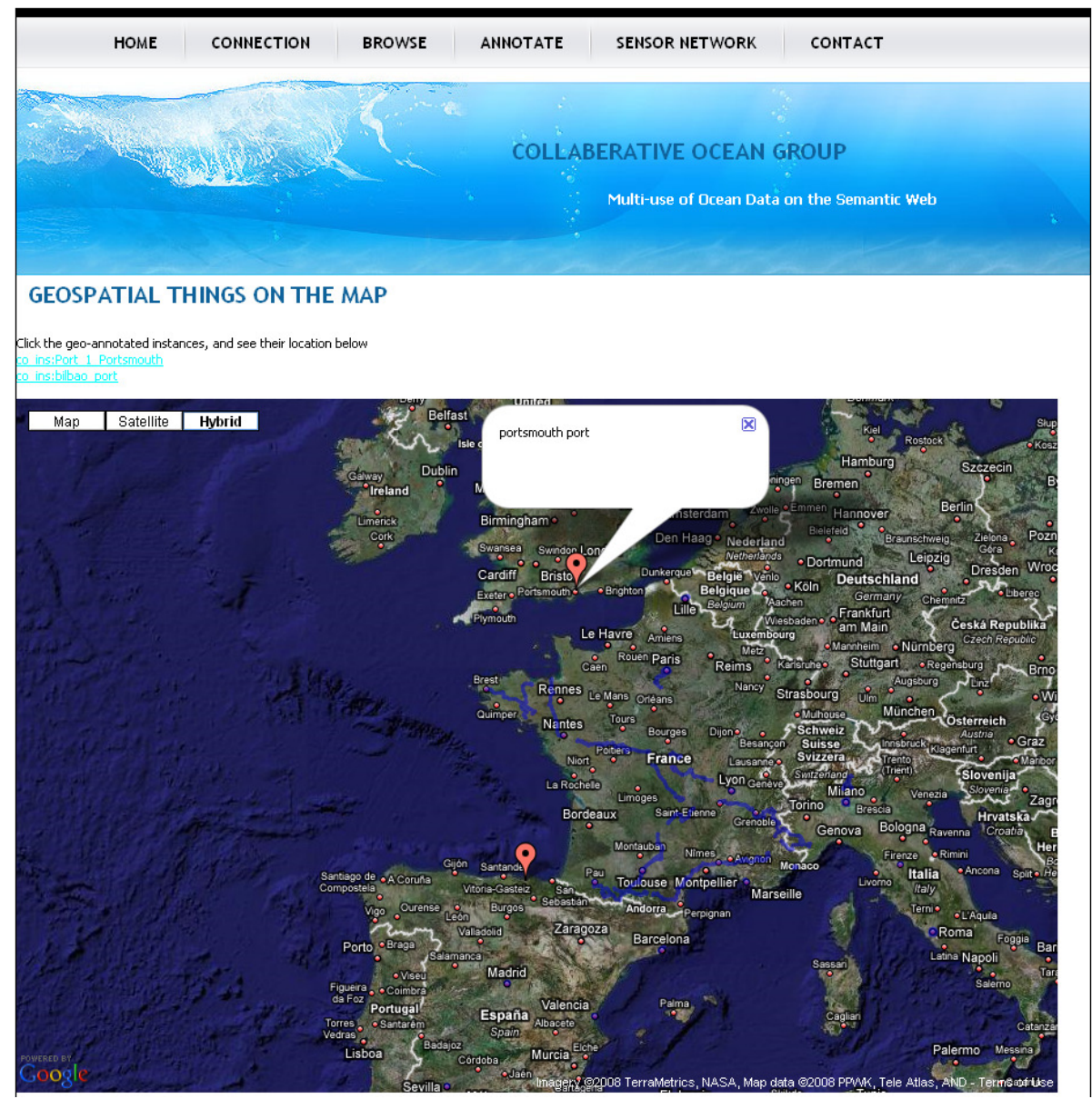

Figure 16 Query and plotting of spatial annotations on the Google map

\section{Discussion and future work}

The Semantic Web technology has moved from a pure research topic to more and more applications in different domains [15] [16] thanks to the growing availability of tools and implementation of W3C specifications. The bottle-neck that prevents abundant publishing of semantic content still lies in the semantic annotation. This is because users tend to hesitate in carrying out the somewhat tedious semantic annotation tasks without being able to see immediate benefit of the input. We believe that this problem can be relieved through two approaches - distributing the task load and batch data conversion.

Since 2002 the data collected from the Pride of Bilbao Ferrybox every 10 minutes has been stored in a database which is used to generate annual measurement graphs shown on the web. However, users or software agents reading the web site have no access to formal descriptions of other related resources that actually collect the measurement datasets. It would be interesting to access such semantic information, e.g. sensor profiles, their calibration and deployment history, from the semantic annotation repository, as they are annotated by their stakeholders in the RDF triple store. This could also help the user to find other similar EO data for comparison by match-making and reasoning their semantic annotations on certain aspects of 
ontological reference model. We also plan to extend the system to a broader context in EO to incorporate more resource annotations into the system.

As can be seen in this paper, there is a clear distinction between ontology and semantic annotation, both of which can be represented in RDF. The ontology, which contains higher level schema-like domain knowledge, has more restricted written access once it reaches a finalized version and is published at a URL. It then serves as a set of formal reference points for definition of all necessary semantics used in the semantic annotations. The semantic annotations are however stored in the triple store which allows more flexible management including modifying, appending, and removing of the RDF triples. This is called the Semantic Web approach. One the other side, for a more open system with less control on the domain schema, ontology can be viewed as having the same level as semantic annotations. That means both of them are stored in the RDF triple store without real distinctions. In this way the ontology can also be modified by end users just as they can do with the semantic annotation triples. This is called the semantic web (lower case) approach. We use the first approach in our work but plan to try the second approach as well for comparison.

With the initial COG infrastructure set up at NOCS, it is now possible to semantically enrich oceanographic resources with ontology based metadata, either defined locally or externally. We have encouraged engineers at NOCS to contribute semantic annotations of their resources in a distributed way and the resulting RDF triples are stored in a persistent storage so that they can be reused by other engineers and data management applications in a later phase.

\section{Conclusion}

In this paper we presented our work on Collaborative Ocean Initiative using the Ferrybox project as a context. The aim is to facilitate resource and data interoperability in oceanographic Earth Observation (EO) to allow multi use of the ocean data. We investigated various technologies and methods, in particular the semantic web approach. By following a semantic web based knowledge life cycle, we have carried out activities on ontology modelling, semantic web annotation of domain resources and their storage as RDF triples and publishing/reuse on the. A semantic web infrastructure has been set up to accommodate these activities. Based on domain knowledge acquired, an ontology based domain reference model has been developed to provide domain conceptualisation and assumptions in the Ferrybox project. Through using different Semantic Web tools and interfaces, we have semantically annotated operational resources such as sensor and platform, as well as observation datasets in the Ferrybox project on the ontology based domain reference model. The ontology and semantic annotations are stored as OWL and RDF triples in a semantic web triple store where they can be further reused to facilitate dynamic ontology driven semantic annotation, linked data browsing, query and data integration.

We have learned through these semantic web activities that the data interoperability problem in EO can be improved by adding a semantic web layer where the ontology based domain reference model is used to

enrich EO resources with standard metadata shared within the community.

\section{Acknowledgements}

This study is supported by the UK Natural Environment Research Council (NERC) through the Oceans 2025 core programmes of the National Oceanography Centre, Southampton.

\section{References}

1. Hydes, David J, Campbell, Jon M, Dunning, Justin, Systematic Oceanographic Data Collected by FerryBox, Sea Technology, Feb 2004

2. Semantic Sensor Web at Wikipedia, http://en.wikipedia.org/wiki/Semantic_Sensor_Web2

3. Amit Sheth, Cory Henson, "Semantics \& Services - Semantic Sensor Web", Published by the IEEE Computer Society 1089-7801/08, 2008 IEEE IEEE INTERNET COMPUTING, Kno.e.sis Center, Wright State University

4. The World Wide Web Consortium (W3C), www.w3.org 
5. MMI-COG, marinemetadata.org/community/teams/cog

6. OpenGIS® Sensor Observation Service Implementation Specification, OGC 06-009r1, 2006-02-13

7. Ferrybox real time monitoring web site: http://www.noc.soton.ac.uk/ops/ferrybox_index.php

8. Hydes, D.J ., Hartman, M.C., Bargeron, C.P., Campbell, J.M., Cure, M.S. and Woolf, D.K. (2008) A study of gas exchange during the transition from deep winter mixing to spring bloom in the Bay of Biscay measured by continuous observation from a ship of opportunity. Journal of Operational Oceanography, 1, (2), 41-59

9. T. Tudorache, N. F. Noy, S. W. Tu, M. A. Musen. Supporting collaborative ontology development in Protege Seventh International Semantic Web Conference, Karlsruhe, Germany, Springer. Published in 2008.

10. TopBraid Composer, http://www.topquadrant.com/topbraid/composer/index.html

11. Auer, S.; Dietzold, S.; Riechert, T. OntoWiki - A Tool for Social, Semantic Collaboration. 5th International Semantic Web Conference, Nov 5th-9th, Athens, GA, USA. In I. Cruz et al. (Eds.): ISWC 2006, LNCS 4273, pp. 736-749, 2006.

Springer-Verlag Berlin Heidelberg 2006.

12. Bo Leuf and Ward Cunningham. The Wiki Way: Collaboration and Sharing on the Internet. Addison-Wesley

Professional, 2001.

13. Ocean2025, http://www.oceans2025.org/

14. Tao, F., Shadbolt, N., Chen, L., Xu, F. and Cox, S. (2004) Semantic Web based Content Enrichment and Knowledge

Reuse in e-Science. In: 3rd International Conference on Ontologies, DataBases, and Applications of Semantics for Large Scale Information Systems (ODBASE), 25-29 Oct, 2004, Larnaca, Cyprus.

15. Tao, F., Millard, D., Zalfan, M., Chen, L. and Davis, H. (2007) Knowledge based Learning Experience Management on the Semantic Web. In: IADIS International Conference of e-Learning, July 6-8, 2007, Lisbon, Portugal.

16. Micah Lewis, Delroy Cameron, Shaohua Xie, Budak Arpinar,ES3N: A Semantic Approach to Data Management in Sensor Networks. Semantic Sensor network workshop, the 5th International Semantic Web Conference ISWC 2006,

November 5-9, Athens, Georgia, USA 2006

17. T. Berners-Lee, J. Hendler, and O. Lassila, "The Semantic Web," Scientific American, May 2001, pp. $34-43$.

18. Fowler, D., Guan, T., Tao, F., Wills, G., Crowder, R., Shadbolt, N.: Comparison of cfd wikis. Technical Report at School of ECS University of Southampton (2008)

19. W3C Linked Data, http://www.w3.org/Designlssues/LinkedData.html 